\title{
Systemic approach to selection-seed-growing sowing with conditional stages of operations
}

\section{Gorobey V.}

Candidate of Technical Sciences

NPO "Selta" National Science Center "Institute of Mechanization and Electrification of Agriculture"

The purpose. To increase efficiency of process of sowing in selection-experimental plots, having ensured certain functional dependences between changeable parameters. Methods. Determination of descriptive and dynamic complexity of system for assessment of amount of elements in system, and diversification of interaction among them. System analysis of component changeable parameters of technique of selectionseed-growing sowing is carried out by targeted change of external actions in entrance point and analysis of responses in exit. Mathematical simulation. Results. For determination of cause-effect relations the seeder is surveyed as a subsystem which most important elements are distributor, drive of distributor and sowing end-effectors. According to process of seeding these elements interact in succession, and alternatives of operation of each of them are surveyed collaterally. Conclusions. The used systemic approach to selection-seed-growing sowing on experimental plots ustifies application of system of electron automatic steering by distributor, combined end-effectors for drill seeding at use of traditional technique, and for broadcasting seeding in soil with minimum cultivation multiple-purpose vomerine nodes which parameters are determined by probed technique, constructive and design-layout parts of seeders.

Key words: seeder, selection, technique, system, process, structure, element, model, vector function, parameter.

Raising of problem. At a comparatively small area (25.100ga) which is taken for seed breedingexperimental works, amount only one row plots in one establishment can make to 70 thousand [1]. The analysis of negative tendencies which take place in the field of selection and seed-growing in the Ukraine allows to make conclusion, that one from reasons of weak competitiveness of domestic sorts, hybrids and seminal products, is there, among other, a low level of technologies and technical providing [5]. A basic problem is related in the production of seed-breeding technique in that in it is needed many different tiporozmiriv machines, and general dsamount each to the type, necessary for the complete providing of requirement in them plant-breeding-experimental establishments, comparatively small at the high cost of machines [6]. For providing of different variants of charts of sowing on the different stages of selection and primary seed-growing must be had sowing machines of different tiposize depending on the amount of seed which are sown, and as a nursery in accordance with the accepted conditional stages selectionseed-growing works. On the first stage of seed-breedings works the exact lay-out of seed is needed through 5 see in a line for sowing of collection and hybrid nurseries, on the second - conduct an ordinary hung posterities of separate seeds or inflorescences (to 500 sht. of seed) on one row or many row plots, to the third - nurseries, which consist of many row plots on which sowing seeds of take, got from the bunch of seeds of the second stage, that in all lines of plot sowing of seeds of the same sort, on the fourth stage carry out an ordinary occupied the seed of, lequminous groats grain-crops on the plots of competitive sort-test of the plots of previous reproduction of new varieties. Research of seed-breedings seeders of different constructions rotined that the model of seeders of the second, third and fourth stages of seed-breeding works can be erected to two basic types after the width of delight: first - with track which changes within the limits of 110-150 see, and second - with track of 160-200sm. Each of these types must include two pidtypes: seeders for sowing nonspaced and seeders for sowing at intervals [2]. Seed- 
breeding seeders of the known producers for researches after modern energysowing technologies are completed, as a rule, by the workings sowing organs of industrial machines, which mass-produce. Domestic seeders, developed from the beginning of 2000th, are tested and made small parties for cereals and lequminous cultures: seed-breeding cassette autonomous distributing for the second stage, sivalka is a cassette central distributing for the third stage and seeder hanging selection-seed-growing for the fourth stage [3] need modernization both on an element base, and structurally for providing of experimental works selectionists at introduction of sorts after new technologies. It is known that traditional development of the separately taken questions in industry of agricultural production is uneffective. Only the analysis of the systems of elements in combination with a mathematical design allows to optimize the system of machines of agricultural production and its application, at maximal reduction of nomenclature of necessary machines and instruments by standardization, universalization, combining, development of variable workings organs, to the base aggregates on the basis of application of compatible technologies [4, 10].

Analysis of the last researches and publications. Very large influence has quality of sowing on the receipt of harvest. For providing of high quality of sowing it is necessary evenly to distribute seed on plots, here a depth of sowing of seed must be identical. The necessary for it plot of feed must be taken every seed. By scientific establishments and long-term economic practice the exactly set amount of seed, which must be sown for the receipt of most harvest. At diminishing, and quite often and at the increase of norm of sowing a harvest goes down. In addition, at a failure to observe of agrotechnical requirements valuable sowing material is unproductively lost $[8,12]$. Sowing of seed on experimental plots appears the most expense operation among other selection-seed-growing works. The method of selection and primary seed-growing foresees comparison of numerous standards of seed or plants of different origin and selection. Standards, which yield to control for qualities of signs which are probed, reject, and the best multiply. With the last conduct subsequent work directly to the transmission of seed in a production. Comparison of standards upon the productivity of separate seeds, after a general harvest from an plot or other useful signs it is possible only at providing of identical terms of their growing . One of important factors there is the adequate placing on plots which are compared. On the first stages of selection (hybrids, mutants, selections, and other), when sowing separate seed and them the first posterities, it is necessary for all seeds to provide the lone person of plot of feed with even geometrical sizes. To the extent of increase of amount of seed in standards, that on the next stages, requirement to exactness of decomposition of seed approach a production. But also here depth of sowing and location of seed in lines, distributing of it on the area of plots which are compared must suit adequacy. Sowing machines in identical terms provide identical quality of placing of seed, but possibility of the use of production machines is fully eliminated in a selection, especially on the early stages and in primary seed-growing [7]. Maintainance of integrity of seed, capacity of sowing of small standards (10-100 grains) for the last grain on certain length of line or with the observance of the set metre norm of sowing, reliability and lightness of cleaning of sowing apparatus from tailings of seed, a complete exception of mixing of standards is an incomplete list of additional requirements. And also, if to take into account that sizes, weight, form and properties of surface of numerous standards of seed, even one culture vary in a wide range, become obvious difficulties which arise up at creation of reliable, outages and enough cheap seed-breeding sowing machine.

Organizationally-technical systems appear dynamic and own properties of adaptiveness, stability, compatibility, and also, in a certain measure, by property of optimization which shows up in adaptation to the environment. By virtue of operating limitations of such systems a tendency takes place to strengthening of optimization, which shows up in the necessity of optimization of structure, functions, minimization of expenses, on development, in growth of efficiency of the systems [11].

Purpose of researches - to promote efficiency of process of sowing on seed-breeding-experimental plots, providing functional dependences are certain between variables by component parameters.

Method of researches. Determination of deskriptive and dynamic complication of the system is for the estimation of number of elements, which are included in the system, and varieties of interaction, 
between them. Analysis of the systems of component in-out parameters of technology selection-seedgrowing sowing conducted by the purposeful change of external actions on an entrance and analysis of reactions on an output. In the technological model of functioning of the system selection-seed-growing sowing indexes of quality of seeds-breedings works on all stages of selection findings closely associated with probability in the possible limits of terms of work and technological modes of operations of seeder. For finding of reasone cocequence connections and mathematical design the seeder is considered as a subsystem by the most essential elements of which, in an aspect there are problems which are examined, there is an occasion of sowing apparatus and sowing worker.

In accordance with the technological process of sowing of seed these elements co-operate consistently, and variants of functioning each of them considered parallell. Developed model of functioning of the system selection-seed-growing sowing creates pre-conditions of determination of technological admittances of quality of work of seeder in the conditions of its normal functioning.

Results of researches. For creation of the system selection-seed-growing sowing structural and functional aspects are taken into account. Above all things, it was presented, as a research model. For researches the systems were taken by indexes which are measured and compared between itself so that possibility to compare between itself the different variants of strategies appeared (methods of achievement of the put purpose). Among the most essential indexes which decided the methods of analysis of the systems: research of efficiency of new methods of occasion of sowing apparatus; development of sowing workings organs; analysis on the indexes of efficiency of interaction of workings organs with new structural decisions with soil after different technologies (hauling resistance, norms of sowing, depth of sowing, but other). Flow diagram of intercommunications of basic component elements in the system of process selection-seed-growing sowing, built on the basic ideas of methodology of analysis [11] of the systems, it is presented on fig.1. Physical properties of seed of agricultural culture and stage of experience (selection or primary seed-growing) appear determining for organizationally technical systems.

Copulas of elements can be one-sided and bilateral, that direct and reverse, when elements influence on each other. System of sowing on plots it is expedient to present as subsystems: seeder and experimental field. The component elements of subsystem seeder is providing of terms for implementation of requirements, above all things, agrotechnical, operating of seeder related to the type for sowing of seed without interval and with intervals, sowing by the apparatus of the autonomous and central distributing of seed, character of his occasion and structural features of sowing working organ. By the staples of subsystem the research field appear accepted in to selection-seed-growing to practice the regulated model of plots for implementation of sowing nonspaced and with intervals, to the process selection-seed-growing work with the width of plots, breadthways spaces between rows, intersowing and between plots paths, breadthways tracks, plots with variables fiziko-mechanical properties of soil. 


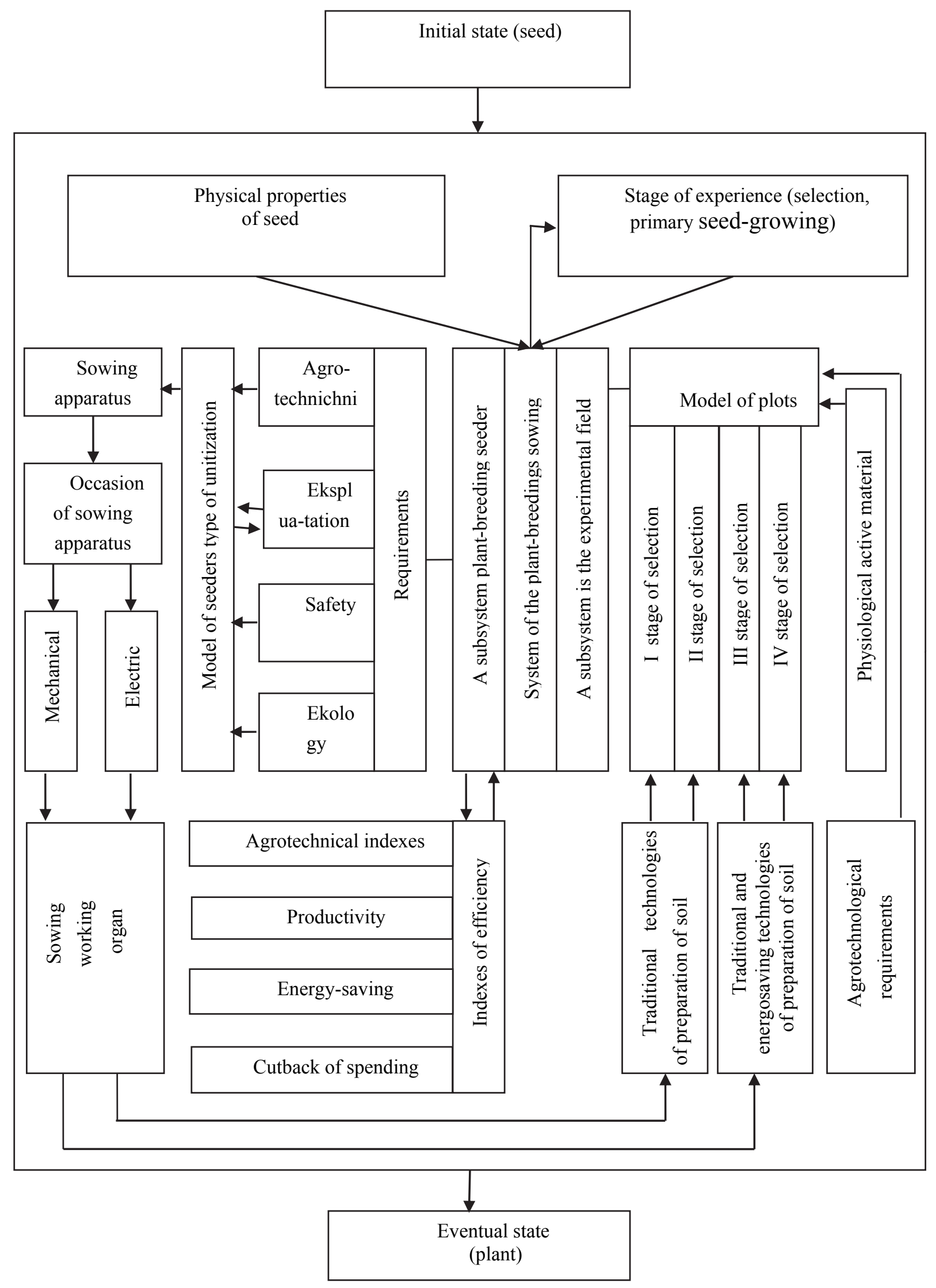

Fig. 1. Flow diagram of system task selection-seed-growing sowing

Considered the application of physiologically active substances, growth regulators, phytohormones, herbicides, retardants, defoliants, desiccants, and regulators of ripening and dormancy of plants and seeds and activators (growth promoters). 
The ordinary process of sowing of seeds in the breeding of agricultural crops is characterized by a large number of variables and parameters is unstable through changes in the uniformity of depth of sowing, physical-mechanical soil properties, limitations, mechanical adjustment of the sowing unit, and other factors. Therefore, the application of automatic control system of seeding, consistent sowing depth with the use of energy-saving factors can increase its effectiveness. The main indicators of the effectiveness of the system processes the selection and seed production of agricultural crops are agronomic indicators of quality, productivity, energy conservation, and cost reduction.

To study the properties of individual elements of the terms of sowing, the system can be considered open.

Thus, it is possible to consider the properties of an element by setting dependencies between the parameters input and output parameters. Consider the model of functioning of a seeder in the form of one subsystem fig. 2.

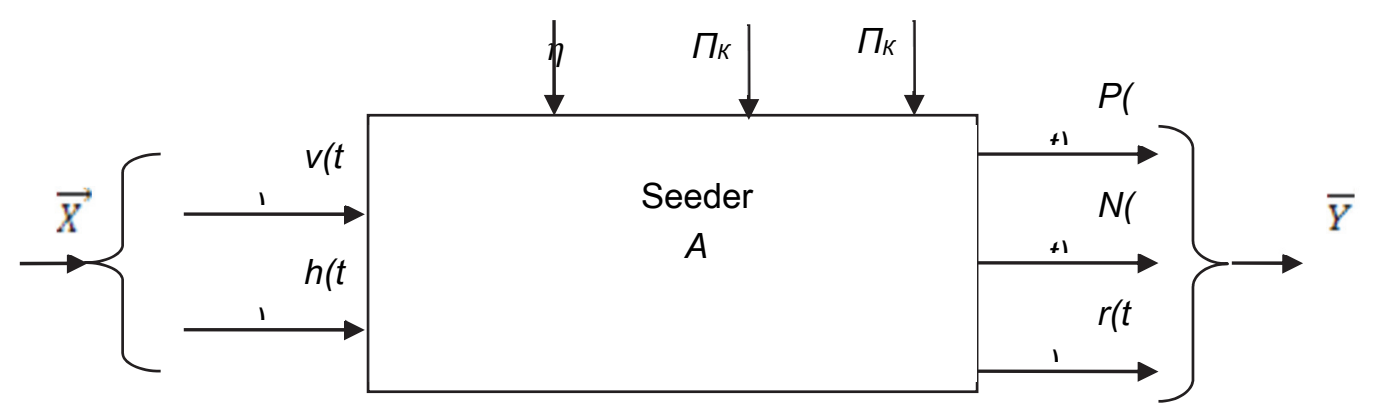

Fig. 2. Functioning model of a seeder in the form of one subsystem

At the input of the model is valid for the vector-function operation modes of the drill, the components of which are the speed $v(t)$, i depth of sowing of seeds $h(t)$. Output parameters represented by the vectorfunction performance seeders: traction resistance $P(t)$, sowing rate $N(t)$, seed germination $r(t)$. Taken into account the gear ratio of the drive of the sowing unit $\eta$, constructive-technological $\Pi_{\mathrm{k}}$ and the kinematic parameters $\Pi_{\mathrm{K} 1}$ seeder. To assess the quality of the seeder scheme of its model augmented vector $=\{P$, $N, r$, which reglamentary operation of the machine.

The essential elements in the seeder, as a subsystem is the drive of the sowing unit, sowing unit sowing working on. In accordance with the process of sowing these elements interact sequentially and functioning of each of them are parallel. For detailed analysis, consider the model of functioning of the drills, presented in three subsystems, each of which has one output $P(t) N(t), r(t)$ and two inputs $v(t), h(t)$ shown in fig. 3. 


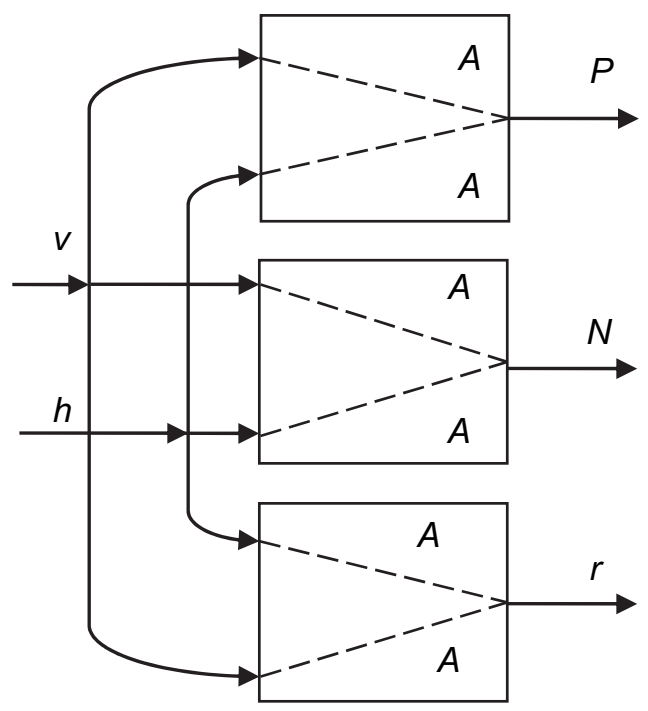

Fig. 3. Functioning model of a seeder in the form of 3 subsystems

Developed a model of functioning of system of seed-growing crops creates conditions determine the process tolerances on the quality of the seeder in terms of its normal functioning.

According to GOST 21878-76, the system operator determines the setting in which each realization of the input signal clearly or interrelation corresponds to the implementation of the output signal. Model function of seeder planter with three subsystems with this definition of the operator we can write the following relationship:

$$
\begin{aligned}
P(t) & =A P^{v}\left[v(t)+A P^{h} h(t)\right] ; \\
N(t) & =A N^{v}\left[v(t)+A N^{h} h(t)\right] ; \\
r(t) & =A r^{v}\left[v(t)+A_{r}^{h} h(t)\right] .
\end{aligned}
$$

To identify the statistical models use regression analysis. Imagine a multi-dimensional model of the seeder in the form of one-dimensional model, using the principle of superposition. The operator $A$ can be represented by the set of partial operators, for the case where the "input" of two variables $v(t)$ and $h(t)$, and the "output" the three - respectively $P(t) N(t), r(t)$ :

$$
A=\left\{A^{(1)}, A^{(2)}, A^{(3)}, A^{(4)}, A^{(5)}, A^{(6)}\right\} . .
$$

So, batometric regression model of the technological process of the seeder with Troma input and output variables can be represented in the form of a system of equations:

$$
\left.\begin{array}{rl}
\mathrm{m}_{\mathrm{P} / \mathrm{v}} & =\mathrm{a}_{\mathrm{vP}}+\mathrm{b}_{\mathrm{vP}} \cdot \mathrm{v} \\
\mathrm{m}_{\mathrm{N} / \mathrm{v}} & =\mathrm{a}_{\mathrm{vN}}+\mathrm{b}_{\mathrm{vN}} \cdot \mathrm{v} \\
\mathrm{m}_{\mathrm{r} / \mathrm{v}} & =\mathrm{a}_{\mathrm{vr}}+\mathrm{b}_{\mathrm{vr}} \cdot \mathrm{v} \\
\mathrm{m}_{\mathrm{P} / \mathrm{h}} & =\mathrm{a}_{\mathrm{hP}}+\mathrm{b}_{\mathrm{hp}} \cdot \mathrm{h} \\
\mathrm{m}_{\mathrm{N} / \mathrm{h}} & =\mathrm{a}_{\mathrm{hN}}+\mathrm{b}_{\mathrm{hN}} \cdot \mathrm{h} \\
\mathrm{m}_{\mathrm{r} / \mathrm{h}} & =\mathrm{a}_{\mathrm{hr}}+\mathrm{b}_{\mathrm{hr}} \cdot \mathrm{h}
\end{array}\right\}
$$

where: $\mathrm{mP} / \mathrm{v} \mathrm{mN} / \mathrm{v}, \mathrm{f} / \mathrm{v}, \mathrm{mP} / \mathrm{h}, \mathrm{m} / \mathrm{h}, \mathrm{mr} / \mathrm{h}$ based on the conditional mathematical expectations of random realization of the input process from the values of the realization of random output process; avP, $a v N, a v P, a h P, a h N, a h r, b v P, b v N, b v P, b h P, b h N, b h r-$ related linear regression coefficients of the mathematical model.

Coefficients of regression equations are determined experimentally to mathematical processing on a PC. The obtained regression model can be used to optimize design parameters selection of seeders and technological parameters of the process of sowing, selection of seeds. 


\section{Conclusions}

Proposed new direction, the essence of which lies in the development and implementation of a system of breeding and seed sowing for the effective use of technological features to create high-performance energy saving selection of seeders. Given a systematic approach to breeding and seed sowing in the experimental plots justifies the application in breeding seeders: electronic autocontrol catoscopium metering device, a combined working bodies for row sowing of seeds in prepared soil according to the traditional technology and roscigno sowing seeds smenarny the cultivation of the soil, the universal ploughshare units that are equipped depending on the requirements of the studied technologies, constructive and structural arrangement of the parts of seeders $[3,9,13]$.

\section{Bibliography}

1. Aniskin V. I. Mechanization of experimental work in breeding, variety testing and primary seedgrowing of grain and bean of cultur/ U. I.Aniskin, J. F. Nekipelov.- M.:NYM, 2004.-200p.

2. Gaifullin, A. H. Unification of the sizes and other parameters of the plots for mechanization of breeding/ A. H. Gayfulin / Breeding and seed production.-1974.- No. 5.- P. 51-57.

3. Gorobey V. P. Selection technique for grain and leguminous crops/V. P. Gorobey, N. Linnik// - Kyiv. "Herald of agricultural science".2012. -№11.- P. 49-53.

4. Drincha V. M. Conceptual and methodological aspects of strategy of development of agricultural mechanization/. V. Drincha.- M.: Russian Academy Of Agricultural Sciences, 2003.-60p.

5. Makrushin N. M. Seed production (methodology, theory, practice): textbook/ N. M. MakrushinSimferopol. ID "ARIAL".- 2012.- 536 p.

7. Mechanization of experimental work in crop production/D..Dunaevsky, E. N. Konyushkov, N.I.Filinkov, V. P. Pyanih - M.:all-Union start.issl. Institute of information and techno-economic research on agriculture.- 1971.- 125p.

8. Mechanization of farming; A.N. Karpenko. - M.: State. ed. agro-literature.-1958.-533 p.

9. Pat. Ukraine, MPK A 01 C 7/00 Seeder mounted selection cassette SNSC-6 / V.P. Gorobey, N. Litvinenko, V. M. Bulgakov, A. M. Macalish, S. S. Starchikov - No. a201405420; Appl.21.05.14;Publ. 25.12.14, bul.№ 24 .

10. Promising directions of scientific researches in the field of agricultural mechanization/ systems analysis in the development of mechanized agricultural technologies: collection of scientific papers/M. S. Ranchev, E. S.Lipkovich, J. M. Siskin etc.// - Zernograd.- FARM, 1984. - P. 3-13.

11. Romanov V. N. System analysis for engineers / V. N. Romanov - SPb: SPbTY.-2006.- 186p.

12. Sycolin P. V. Tillage and sowing machines: history, engineering, design /P. V. Sycolin, L. V. Pogoriliy.- K.: Phoenix, 2005.- 264p.

13. The theoretical basis of the oscillation of gear selection opener/U. V. Adamchuk, V. M. Bulgakov, I. V. Golovach, V. P. Gorobey//Mechanization and electrification of agriculture. - Vol.1(100).-Glevaha.2015. - P. 10-21. 